\title{
Predictive Model based on Sentiment Analysis for Peruvian SMEs in the Sustainable Tourist Sector
}

\author{
Gianpierre Zapata ${ }^{1}$, Javier Murga ${ }^{1}$, Carlos Raymundo ${ }^{1}$, \\ Jose Alvarez ${ }^{2}$ and Francisco Dominguez ${ }^{3}$ \\ ${ }^{1}$ Escuela de Ingeniería de Sistemas y Computación, Universidad Peruana \\ de Ciencias Aplicadas (UPC), Lima, Lima, Perú \\ ${ }^{2}$ Departamento de Informática, Universidad Carlos III, Getafe, Madrid, Spain \\ ${ }^{3}$ Facultad de Informática, Universidad Rey Juan Carlos, Mostoles, Madrid, Spain
}

Keywords: Sentiment Analysis, Big Data, Cloud Computing, Travel Management Process, Tourism Sector.

\begin{abstract}
In the sustainable tourist sector today, there is a wide margin of loss in small and medium-sized enterprise (SMEs) because of a poor control in logistical expenses. In other words, acquired goods are note being sold, a scenario which is very common in tourism SMEs. These SMEs buy a number of travel packages to big companies and because of the lack of demand of said packages, they expire and they become an expense, not the investment it was meant to be. To solve this problem, we propose a Predictive model based on sentiment analysis of a social networks that will help the sales decision making. Once the data of the social network is analyzed, we also propose a prediction model of tourist destinations, using this information as data source it will be able to predict the tourist interest. In addition, a case study was applied to a real Peruvian tourist enterprise showing their data before and after using the proposed model in order to validate the feasibility of proposed model.
\end{abstract}

\section{$1-$ INTRODUCTION}

In recent years tourism has become a powerful transformative force that has had a decisive influence on the lives of thousands of people. This is because it is one of the main employment generation sectors in the world (Scowsill, D., 2017) being the sector that presented a growth of $3.1 \%$ in 2016 , it contributes a 9.8\% to the Gross Domestic Product (GDP) worldwide. That is why the United Nations (UN; 2015) declares the year 2017 as the International Year of Sustainable Tourism for Development, seeking to encourage a change in policies, practices of tourismrelated businesses and to evaluate consumer behavior in order to promote a more sustainable tourism sector.

One of the limitations of the current tourism model is associated with the progressive growth of productivity in the international market, facing important challenges that emerge from the need to reestablish its comparative advantages over other competing destinations / countries. Faced with this need, the search for new innovative solutions, understood as new products, products, processes, new marketing techniques or organizational improvement to minimize costs and to differentiate the product of the offered service and, ultimately, target all these strategies to increased productivity of the system is underway. In several tourism subsectors there is a rapid process induction to new innovation technologies that are changing the bases of production and market structure. Without deviating, the tourism sector seeks to evaluate consumer behavior in order to be able to make decisions, so it has taken an increased interest to exploit the countless amount of data generated by the social networks, where users shed their unbiased opinions of any subject (Thomas H. Davenport, 2013).

However there are websites that try to cover the chain value by providing services based on an information catalog and supported by previous opinions and experiences as seen on TripAdvisor, Booking, HotelsCombined, Agoba, Kayak, among others. But these platforms cannot measure the interests of consumers and in a market where international competition is growing, a tourism forecasting model must be able to deal with the development of competitive advantages which will allow a better performance to select a tourist destination. 
In this sense, we propose a tourist interest prediction model based on the sentiment analysis of social networks and their results. This research is divided into the section that describes the research that are the basis for the proposed model, modeling, implementation results, and conclusions.

\section{BACKGROUND}

\subsection{Sentiment Analysis}

Sentiment analysis refers to the use of word processing and analysis tools to quantify the sentiment expressed in words. This analysis helps discover trends and reflect the real world in social networks (Kiran Garimella et al., 2016). It also helps predict different topics, in this case the tourist traffic and time series of social networks.

In order to carry out this analysis, one can proceed in different ways, within which one is making the analysis based on a lexicon another being the ontological analysis (Ali Marstawi et al., 2017). In the first type of analysis, the classification is based on the present words and the number of occurrences, taking into consideration the semantic orientation of the words, which must be added to a dictionary classifying and rating them in negative and positive, according to their intensity level (Anna Jurek et al., 2015). In the ontological analysis a model is generated by classifies the feelings based on concepts (Pratik Thakor et al., 2015).

It is clear that the sentiment analysis is a discipline that is generating interest in the scientific community, resources have been produced such as Word2vec (Dongwen Zhang et al., 2015), WordNet Affect, SentiWord Net, among others (R. Linares et al., 2015), which allows the generation of new research cases and then a predictive analysis to examine in this case the tweets and be able to analyze the origin location, if it is classified in a positive or negative way, among others established parameters (Eric Baucom et al., 2013).

Thus, observing case studies is revealed that this sentiment analysis allows us to make a prediction on a specific topic as the case of the stock market analysis, where values can be predicted according to consumer trends (John Kordonis et al., 2016). This allows us to affirm that this analysis is scalable to diverse scenarios like the tourism sector.

\subsection{Prediction Model}

A predictive model is a way of observing the data that one has, classifying it and then after an analysis, being able to predict, based on the chosen parameters, a future result. To do this, the data has to be parameterized according to certain features chosen according to the characteristics to be predicted. In order to succeed, we must have the precise number of variables, if insufficient variables are specified, the model produces partial estimates, if there is an excess of variables the model produces low accuracy estimates. These variables will be defined according to the chosen field, in this case study will be related to tourism.

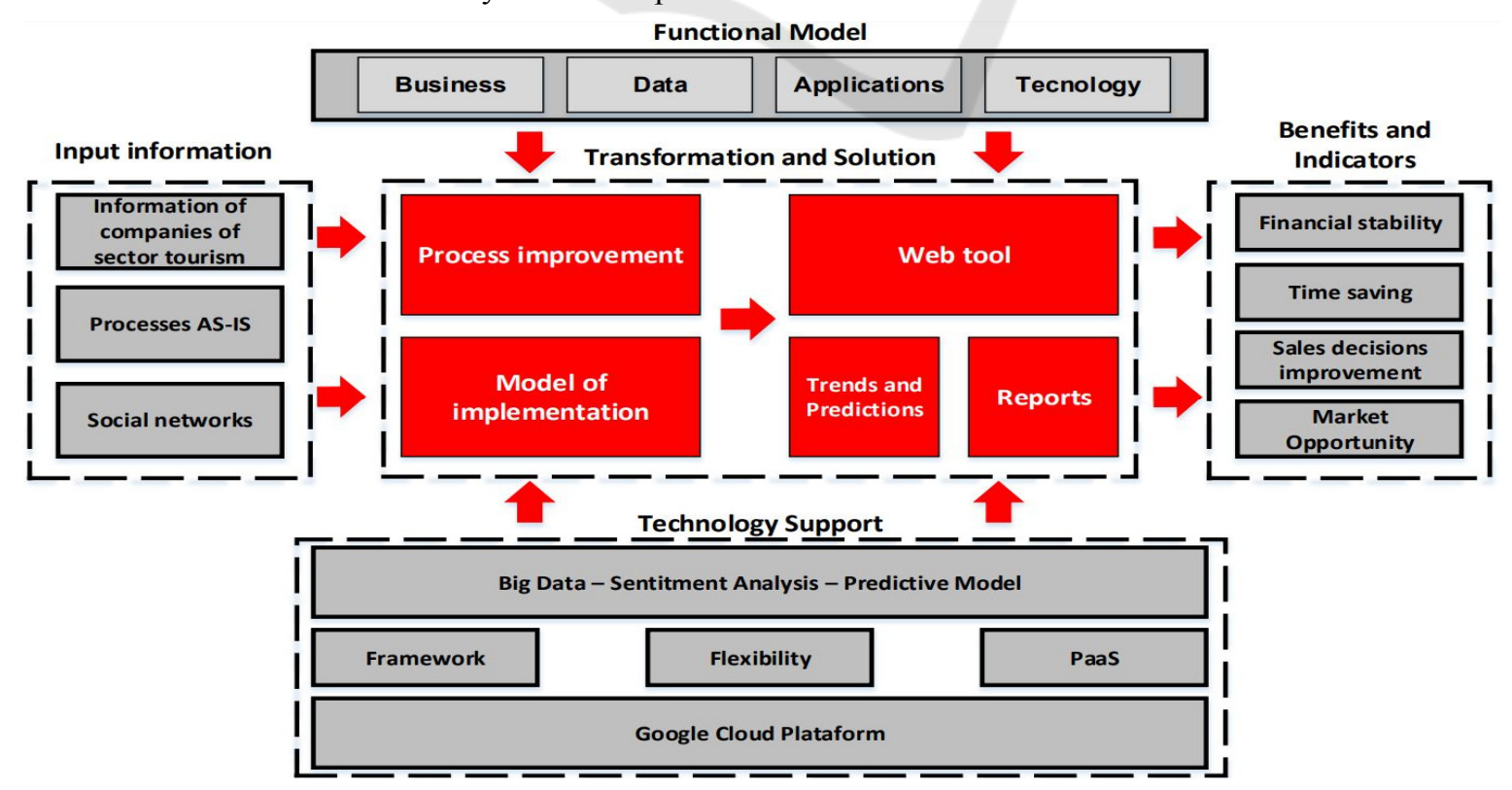

Figure 1: Technological Model. 
The consulted research, shows a relation between social networks and the information they store, which gives us the ability to predict events, such as presidential elections, tourism expectation or sales of products. In addition, in a case study it was possible to demonstrate how the power of social interactions in the form of an advertising campaign and the tweets generated by it can be analyzed to predict to a certain extent the results of a future presidential campaign (Andranik Tumasjan, et al. 2010).

Therefore, we can say that the expectation has been seen as a point of reference that consumers use to determine the satisfaction or assessment for the performance of products or services (Chunyang Wang et al., 2016), but it is contrasted with how the expectation is related to the result of a number of factors generated in the particular case of a film shown in cinemas (Yang Liu et al., 2007). These statements generate a motivation about tourism, which can be classified into two forces that describes how individuals are motivated to make a decision, in this case a trip and how they are attracted to a particular destination by its qualities. On this subject, the image that each individual has of the destination is closely related to the motivation he has to travel to that particular place (Chunyang Wang et al., 2016).

\section{PROPOSED MODEL}

\subsection{Analysis of the Model}

According to the research that was carried out for the development of the model and based on previous researches that presents scenarios where the use of the internal processes of the tourism sector can be used as sources of information that allows the analysis of the characteristics and behaviors of consumers (Carlos Raymundo et al., 2017), a need was found in the tourism sector. Thus the reason a user interest prediction model is proposed. Under this approach, we asked the following question: What are users looking for? This can be answered by enhancing its decisionmaking management and in the future to predict tendencies according to the interests of the users.

Consequently, a model for predicting the tourist interest is proposed as it helps to significantly increase the decision making capacity. The main idea of the proposal is to develop a model that has technological aspects as well as to improve the process of travel management by adding a new channel to existing activities. It should be mentioned that the prediction model is part of the technological mode. Taking in consideration that in order to develop it first one must know the business and after making the process improvements, propose a technological solution that meets the need. The model presents 5 components: information input, functional model, transformation and solution, technological support and lastly benefits and indicators (See Figure 1). With each one being able to retrieve the necessary information from the sector and recognize the needs of the business, to later define the company's processes and identify opportunities for improvement.

Based on this analysis, it is possible to define an implementation model, one that can identify benefits and business indicators. Finally, to improve processes and automate them through a technological solution that allows analyzing the information of social networks' history and it's in real time and processes to generate new business opportunities.

\subsection{Components}

\subsubsection{Input Information}

Although companies in the tourism sector have limited capacity in their resources, as well as its processes, each has its own complexity and information extraction mechanisms (be it quantitative or qualitative). For this reason, some of the types of information needed to have a general framework with respect to tourism companies are shown, including information on the current situation of companies in the tourism sector that allow the identification and processes As-Is, in addition to looking for a way to solve the problems that arise in the sector. Therefore, an external investigation was carried out in order to understand the following question: What is the most commonly used information search channel? A survey was carried out with a sample of 300 users distributed in 4 well-defined groups and organized by age ranges, from 17 to 24 years, from 25 to 40 years, from 41 to 60 years and 60 from more. The group with the highest percentage as shown in Table 1 is the one that includes ages from 25 to 40 years with $40 \%$. Taking into account that $55 \%$ are male and $45 \%$ female.

The search channel type resulting of the surveys is demonstrated in a ranking Table 1.

Table 1: Search channels ranking.

\begin{tabular}{|cc|}
\hline Search cannel type & $\mathbf{\%}$ \\
\hline Social Networks (Facebook, twitter, youtube, & $46 \%$ \\
among others). & \\
Travel and Tourism Companies & $29 \%$ \\
Friend recommendation & $16 \%$ \\
Newspapers and magazine & $7 \%$ \\
Other & $2 \%$ \\
\hline
\end{tabular}


Being the main interest of this study to investigate the trends of travel and interest of users, the $46 \%$ of the interviewed assess their travelling options with social networks as a search channel. As a result, companies in the sector can use social networks as a new way for collecting information and making decisions based on the needs and trends of the user.

\subsubsection{Functional Model}

Each company in the tourism sector is different and has a complexity of its own according to its structure, but all of them have shared aspects. That is why the best practices can be gathered to model their processes, data, applications and networks. The functional model presented is segmented to have the ideal processes in terms of travel management, taking into account the addition of a new information search channel. Thus, in the event that at the moment of releasing information from the tourism sector the company does not have the defined processes, the functional model can be supported to fill that gap with a structured and defined process for them, in addition to contemplating all the data and documents to determine the improvement of the processes of the company. With regard to networks and applications, it is the reflection of how to model these aspects that will be modeled in the next stage.

\subsubsection{Technological Support}

The technological model is determined by several levels as is grouped by applications and deployment or networks. In turn, these are classified by Application Servers, Database, Operating Systems and Cloud Services (See Figure 2).

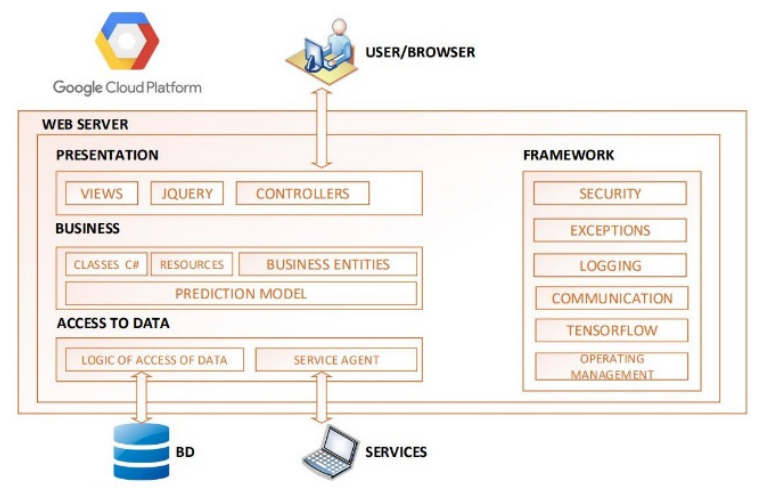

Figure 2: Deployment model.

The levels shown in this Technological Support component are detailed from the bottom to the top, since part of the need for a solution to be available in the required time and at the same time that the costs associated with the solution are adequate to the situation in which the company in the sector is due to its limited purchasing power. The levels of the component are as follows:

Google Cloud Platform: Due to the situation that companies in the tourism sector are found, it is proposed the use of Cloud Computing services as a lower level of infrastructure. Within the cloudoriented services there are types, which are: Software as Services (SaaS), Platform as Services (PaaS) and Infrastructure as Service (IaaS). For this reason, the PaaS was chosen because of the need for a platform to work on the development of the solution.

Likewise, we performed an analysis among the other cloud service providers where we found wellknown companies as Azure, AWS, IBM SoftLayer and Google. Among the aforementioned, Google Cloud Plataform (GCP) it's starting to have a greater impact on the market as it has cheaper tariffs and the ability to compete with the other suppliers. In this sense, for this contribution, this provider is taken and services called Cloud DataStore API and Machine Learning Engine.

Framework, Flexibility and PaaS: For the development of the system we use the ASP.Net MVC 4 framework, which provides a suitable development environment for the application, since we can clearly separate the data loads and use of them to be displayed in the application. The flexibility is found in the cloud platform, as it is scalable in nature, it adapts the ingestion of data loads, either little or if need be, massive amounts of data. The ability to perform this task was of vital importance to be able to build the system, since it adapts to variable data loads. Within the cloud services we choose PaaS, since the model covers the entire development cycle from planning to implementation and testing. It was also used because it reduces the costs of maintenance, having a constant monitoring tool and the greater availability of service use.

The application was developed based on these three concepts, the framework gives us the ease to develop and maintain; PaaS is in the integration of database services, sentiment analysis and continuous deployment and the flexibility is found in the platform capacity to deal with variable amounts of data.

Big Data - Sentiment Analysis: To know the interest of the user in the places expressed in the Tweets it was decided to carry out a sentiment analysis, which was carried out through the Google Prediction API. For the training of the model a package of 379 examples was used for the classification in four 
categories, good, regular, tedious and bad. With this information we can know in detail the user's feeling. Data extraction and analysis was performed following the steps of the following illustration (See Figure 3).

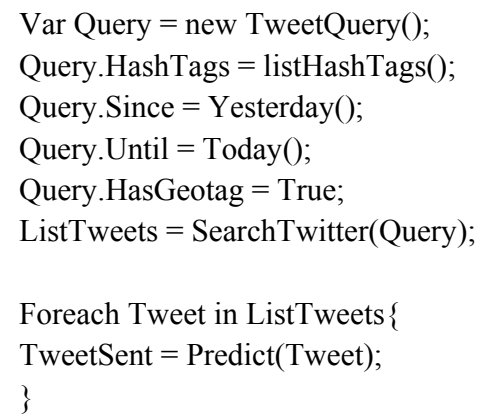

Figure 3: Load and analysis pseudo-code.

The terms used in the pseudocode for extracting data from Twitter and the subsequent sentiment analysis are as follows. A Tweeter query object is initialized, to which the search parameters are assigned. In the case of the project, a list of HashTags related to travel and tourism was used, as the program has a period of periodic execution, the extraction of tweets was limited from the previous day to the present day, and finally, to determine the place from where the tweet was launched, a flag was used to collect only tweets containing geo-location. Once collected, the list is sent to the Google Prediction API where the sentiment analysis is performed and returns the tweet's rating as a response.

Prediction Model: To create the prediction model, a neural network of the Deep Feed-Forward type was used to predict the tourist interest. The neural network, takes the input data in the first layer and after being classified by the hidden layers of the network, returns the result that was defined according to the model. This model was trained with data from the sentiment analysis to have a more accurate prediction. Taking into consideration that for the development the wide and deep models were joined, this gives the neural network a high capacity of abstraction (See Figure 4). In the proposed model, horizontal characteristics are taken as day or country, in addition to using cross features to give the model more certainty. Within the vertical characteristics were taken the entry columns. These models are linked to the data output of the linear regression of the DNN * (Deep Neural Network). This union gives us speed and accuracy of calculation, with which we could predict the tourist interest.
InputColumn = CSV_Column (activity, day, place,

country);

WideColumn $=$ [crossed (activity, place), day, country $]$

DeepColumn $=$ [activity, day, place, country $]$

DNNLinearCombinedClassifier(WideColumn,

DeepColumn )

Figure 4: Neural network pseudocode.

\subsubsection{Transformation and Solution}

This component will take as input the processes AsIs improved and adapted to what is collected from the functional model. At this point, To-Be processes are defined which are recognized as the final processes. It should also be taken into account that these processes have process improvements by adding the proposed new channel, an implementation model to be developed in the company and the web tool that supports it.

\subsubsection{Benefits and Indicators}

Then the section of Transformation and Solution, should have results that make the tourism company more efficient, so we detail the following benefits and indicators of success:

Financial Stability: This indicator will allow the company to control its expenses, besides allowing to negotiate discounts with supplier companies by having a clear idea of the consumers need.

Time Saving: This indicator will reduce the sales decision making and creation of tourism packages time, because it will allow to visualize the need of the consumer in the system.

Sales Decisions Improvement: This benefit allows the company to visualize forecasting analysis of the user interest so that decisions can be made in a shorter time without depending on the companies that control the market.

Market Opportunity: This benefit is the most important, because having a new information search channel will give the company means to compete against large companies by having real-time information on the consumer's needs.

\section{VALIDATION}

To validate the presented prediction model, we will use a case study to show that the proposal successfully solves the needs of tourism companies. 
It can be said that it is validated as a part of the model in order to ensure its correct functioning in the case study. The components involved will be transformation and solution for the practical validation through the case study. In addition, it is mentioned that for confidentiality purposes the real company of the sector will be represented as OT S.A.C.

\subsection{OT S.A.C.}

OT S.A.C is a small company dedicated to the sell and distribution of tourist packages, a business that has 10 employees and a monthly turnover of approximately $\$ 29,447.85$. It is located in the district of Santiago de Surco, in the city of Lima, Peru. Its main suppliers are the wholesale companies in the sector, which give a list of packages for sale and distribution. In turn, this company sells custom packages according to customer's requirement.

This company, since its beginnings, according to the national regulations regarding the package distributors, was favored by the client portfolio that already had because of related businesses. But as new competing companies appeared in the sector with same products, it led to a price contest that gave a sudden growth of companies in this area.

Under this circumstance, the company owners and managers focused on the vision of using new technologies and trends to keep the company's sales afloat. That is why it is proposed to this company to implement a prediction model in collaboration with it's the workers, as well as the direct managers of the company.

\subsection{Implementation}

The schematic to implement the proposed model can be visualized in Figure 5, which details the necessary steps that the company OT S.A.C has to implement for this proposal, the graph is read from top left to the bottom right of the graph.

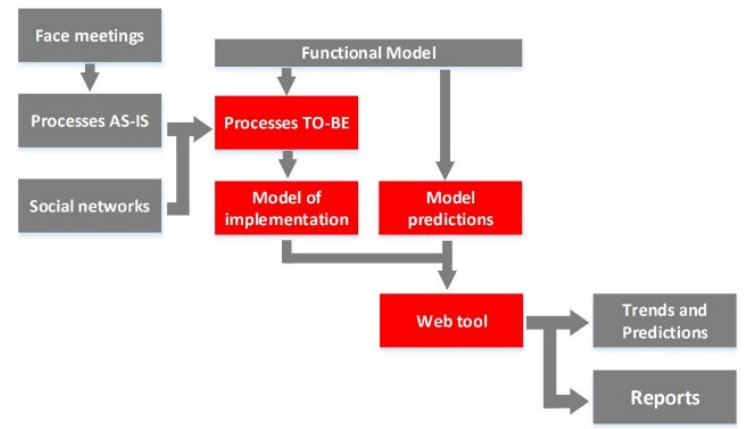

Figure 5: Technological model implementation.
Face Meetings: The implementation was made through weekly meetings with the owners and workers involved, during this period were explained the company processes. OT S.A.C has the following processes:

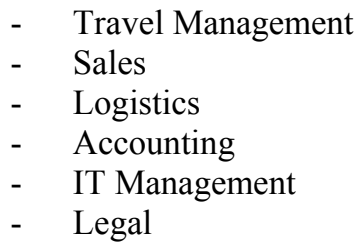

Processes As-Is: When carrying out an information survey, it was noted that the company OT SAC did not have well-defined processes, so it was not possible to design an As-Is, but it was based on all the functions performed by each worker to recognize their activities and then the processes they performed.

In the process of developing processes As-Is, we detect a dependency in wholesalers companies that control the market, so that a new information collection channel is presented where companies can make decisions based on the needs of users and trends shown on social networks.

To-Be Processes: With the identified processes, a detailed work was done to be able to define which key processes the company should carry out to have an optimal performance, without any voids that can affect it. Reason why it was based on the functional model to reference the components and thus be able to contrast their information gaps.

In this particular case, we identify the following wrongdoings inside the Travel Management process.

- The process does not have proper documentation or any additional information other than the mayor market controlling companies.

- The input information they have is outdated, as is based on the historical record of other companies.

- The user requirements response time is limited to the response that can be provided by another company that has the information.

These are the main findings compared to the Functional Model. To solve these problems we introduced a new information search channel within the process supported by an application developed for the company. In this way the company can make realtime decisions according to the user's tendencies and the predictions that tool generates.

Implementation Model: It is the graphic representation of the phases that the company must carry out in order to implement the proposed model. Taking into account that training is planned in the company to 
perform a correct management of the organizational change and prepare the workers to use the new tools and technologies in this sector.

Predictive Model: Taking into account the benefits of implementing a sentiment analysis along with a predictive model, the solution was implemented in the company OT S.A.C. To implement the model, the training was carried out with test data from the sentiment analysis. Figure 6, shows the learning process of the neural network through each iteration. Once a job is sent to train the neural network with the model, it makes use of the neurons to be able to infer the results. The progressive increase of accuracy in each step of the training phase, demonstrates the learning capacity of the network.

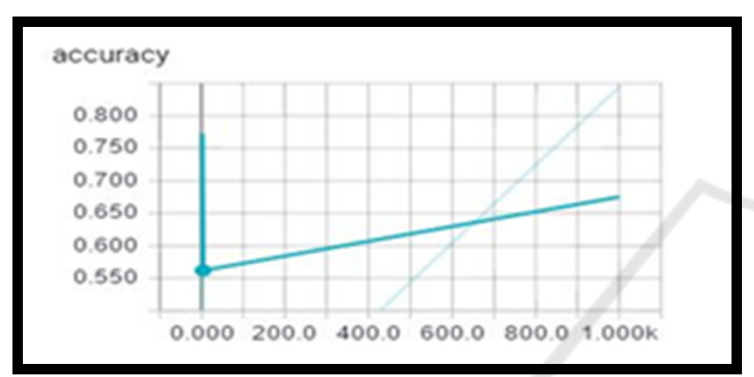

Figure 6: Accuracy graph.

Web Tool: To develop the prediction model for the company OT SAC, a web tool was devised in order to research and collect information from a social network, in this case twitter. This information will analyze the users' comments and identify their preferences, tastes and mood, etc. This will provide rating information of a city, country and travel package, as well as to generate input data to subsequently predict travel destinations with a greater acceptance range.

For this part, we used the concepts of Sentiment Analysis presented in section 3.2.3; which allowed us to analyze the tweet of users worldwide. This helped us to create a database with the following fields ID, Activity (Museum, Beach, Trekking, Adventure Tourism), Country, City (Place), Year, Day, Month, Range (Good, Bad, Regular, Tedious) and finally the tweet itself to analyze. (See Figure 7a and 7b).

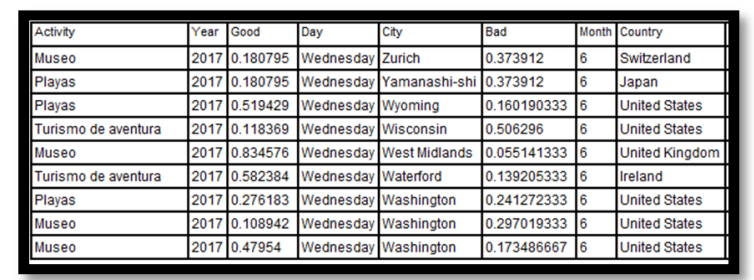

Figure 7a: Database of analyzed tweets. Part 1.

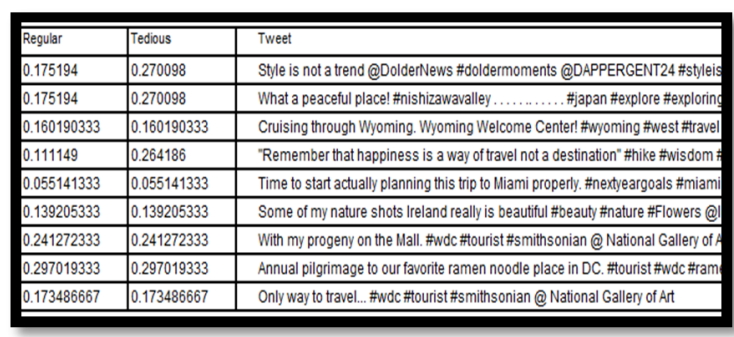

Figure 7b: Database of analyzed tweets. Part 2.

With these results and using the prediction model integrated into the web tool, it is possible to predict the trend and activities the users will have in different parts of the world (see Figure 8). In addition, we can identify which days are highest rated among users to plan a trip (see Figure 9). Taking it into account, a metric to analyze this information was constructed, dividing it into three categories as described in the following lines, good, average and bad, as seen in Table 2. Furthermore it grants the user a view of the raked packages from other users. (See Figure 10).

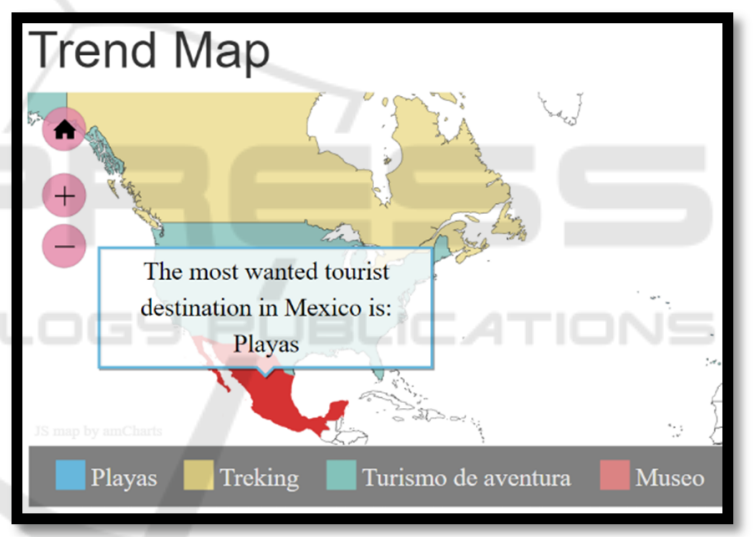

Figure 8: User tendency map.

Table 2: Ranking metrics by city, country or package.

\begin{tabular}{|c|c|}
\hline Metric & Category \\
\hline$[0-0.25]$ & Bad \\
\hline$[0.251-0.75]$ & Regular \\
\hline$[0.751-1]$ & Good \\
\hline
\end{tabular}

\begin{tabular}{llll}
\hline Results & & & \\
City & Day & Score & Mark \\
Florence & Wednesday & 0.795802 & Good \\
Florence & Saturday & 0.85798753125 & Good \\
Florence & Sunday & 0.86402725 & Good \\
\hline
\end{tabular}

Figure 9: Rating reports by city. 


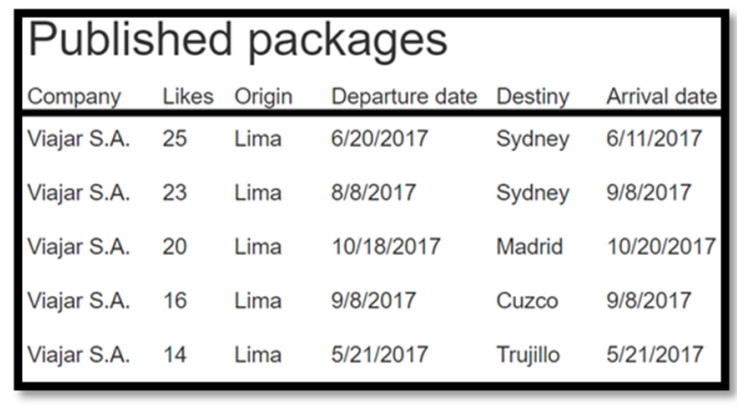

Figure 10: User trends map showing activities by country.

\subsection{Results}

Once implementation of the technological model in OT SAC was completed, it can be observed that the benefits and indicators in the processes using the new trends and technologies assure a greater quantity of sales. It is also considered that using tools of massive data analysis, in accordance to International Data Corporation (IDC) (Carlos Raymundo et al., 2017), progressively increase sales by a $12 \%$ margin. In order to test this assertion, an analysis of the loss indicator due to logistical expenditures was made, in Fig. 15. It presents all the logistical movements that are considered expenses. The $57 \%$ of the total annual expense and costs are due to two mayor causes. The first cause is the difference in purchase prices, amounting to $17.6 \%$ of the total. The other reason is the unnecessary purchases taking a $39.9 \%$ of this total. After the implementation of the technological model and thanks to the support provided by the prediction model, the company OT S.A.C was able to reduce their logistical expenses as explained in Table 3. Considering a $40 \%$ threshold.

Table 3: Loss caused by logistical expenses.

\begin{tabular}{|c|c|c|c|}
\hline \multicolumn{4}{|c|}{ Loss caused by logistical expenses (\$) } \\
\hline \multicolumn{2}{|c|}{ Before } & \multicolumn{2}{|c|}{ After } \\
\hline $\begin{array}{l}\text { Annual } \\
\text { expenses } \\
\text { cause by price } \\
\text { difference. }\end{array}$ & 4824.24 & $\begin{array}{l}\text { Annual } \\
\text { expenses } \\
\text { cause by price } \\
\text { difference. }\end{array}$ & 3600 \\
\hline $\begin{array}{l}\text { Annual loss } \\
\text { cause by } \\
\text { purchases. }\end{array}$ & $11,104.3$ & $\begin{array}{l}\text { Annual loss } \\
\text { cause by } \\
\text { purchases. }\end{array}$ & 7773.01 \\
\hline Loss $\%$ & $57 \%$ & Loss $\%$ & $27 \%$ \\
\hline Indicator & Critic & Indicator & Positive \\
\hline
\end{tabular}

Upon a closer inspection of the results, the following can be said:

- Before the implementation of the technological model, the company had a large margin of loss in the purchase and separation of packages. The reason for this behavior can be explained because the company did not have a clear reference on the need of its customers. Now with the model, not only can company recognize the customers' need, it can also negotiate with its suppliers new rates that will generate a higher gross sales income.

- The feasibility study (see Fig. 11), shows the investment and recovery periods projected after the model implementation. It shows a \$ 24,212.92 investment over 12 month. It includes both the workforce cost for the implementation and cloud services costs. Culminating that period the only cost would be the cloud services. At this time frame the company will have an estimated profit of $\$$ $25,953.93$ due to insured sales. These last values are made possible by having an investment return of approximately 11 months, with a ROI of $114 \%$, being totally profitable for a company in the tourism sector.

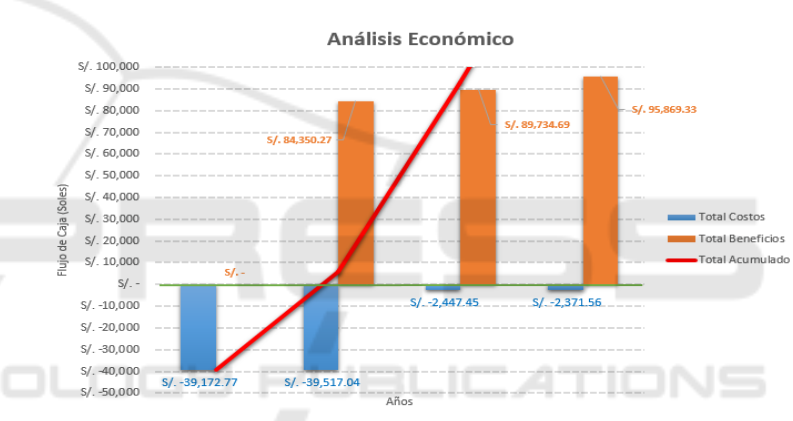

Figure 11: Feasibility study.

- We arrive at the conclusion that the joint implementation of a technological and predictive model will bring benefits to the tourism sector as it generates new market opportunities.

\section{CONTINUITY}

In future proposals new instances of the business model could be applied and be escalated across the company, not only generating new sales business channels, allowing us to make more accurate decisions, but it could also be used in other administrative process, reducing their cost.

In addition, it opens a myriad of possibilities for related and unrelated fields where the analysis of massive social network data could reduce the operating costs and increase the company's revenue. 


\section{CONCLUSION}

To answer the problem of controlling the logistical expenses based on a technological model through a reengineering, process improvement, the use of Cloud Computing and Big Data is needed to support a company in the tourism sector and to obtain both financial and operational stability.

It was demonstrated that the use of technologies such as Cloud Computing and Big Data oriented in a free software guideline is reliable for companies in the tourism sector, since the cost of these services are adequate to the purchasing capacity of small and medium enterprises.

Finally, it was demonstrated that a control vision provides utilities for an industry in an indirect way, reducing the losses in logistical expenses, saving time creating packages and money as a result.
Dongwen Zhang, Hua Xu, Zengcai Su, Yunfeng Xu, Chinese comments sentiment classification based on word2vec and SVM, Expert Systems with Applications, Volume 42, Issue 4, 2015, Pages 1857-1863, ISSN 0957-4174, http://dx.doi.org/10.1016/j.eswa.2014.09. 011

R. Linares, J. Herrera, A. Cuadros and L. Alfaro, "Prediction of tourist traffic to Peru by using sentiment analysis in Twitter social network," 2015 Latin American Computing Conference (CLEI), Arequipa, 2015, pp. 1-7. DOI=https://doi.org/10.1109/CLEI. 2015.7360051

Eric Baucom, Azade Sanjari, Xiaozhong Liu, and Miao Chen. 2013. Mirroring the real world in social media: twitter, geolocation, and sentiment analysis. In Proceedings of the 2013 international workshop on Mining unstructured big data using natural language processing (UnstructureNLP'13). ACM, New York, NY, USA, 61-68. DOI=http://dx.doi.org/10.1145/2513549. 2513559

John Kordonis, Symeon Symeonidis, and Avi Arampatzis. 2016. Stock Price Forecasting via Sentiment Analysis on Twitter. In Proceedings of the 20th Pan-Hellenic Conference on Informatics (PCI'16). ACM, New York, NY, USA, Article 36, 6 pages. DOI=https://doi.org/ $10.1145 / 3003733.3003787$

Scowsill, D., 2017. Global Economic Impact \& Issues 2017. Economic impact research. World Travel \& Tourism Council. Retrieved June 9, 2017 from https://www.wttc.org/-/media/files/reports/economicimpact-research/2017-documents/global-economicimpact-and-issues-2017.pdf

UN, 70/193, General Assembly, 70th Sess, Supp No 81, UN Doc A/RES/70/193 (2015)

Thomas H. Davenport. 2013. At the Big Data Crossroads: turning towards a smarter travel experience.

Kiran Garimella, Gianmarco De Francisci Morales, Aristides Gionis, and Michael Mathioudakis. 2016. Quantifying Controversy in Social Media. In Proceedings of the Ninth ACM International Conference on Web Search and Data Mining (WSDM '16). ACM, New York, NY, USA, 33-42. DOI: https://doi.org/10.1145/2835776.2835792

Ali Marstawi, Nurfadhlina Mohd Sharef, Teh Noranis Mohd Aris, and Aida Mustapha. 2017. Ontology-based Aspect Extraction for an Improved Sentiment Analysis in Summarization of Product Reviews. In Proceedings of the 8th International Conference on Computer Modeling and Simulation (ICCMS '17). ACM, New York, NY, USA, 100-104. DOI: https://doi.org/ $10.1145 / 3036331.3036362$

Anna Jurek, Maurice Mulvenna, Yaxin Bi, Improved lexicon-based sentiment analysis for social media analytics, Security Informatics, Volume 4, 2015, ISSN 2190-8532, http://dx.doi.org/10.1186/s13388-0150024-x

Pratik Thakor, Sreela Sasi, Ontology-based Sentiment Analysis Process for Social Media Content, Procedia Computer Science, Volume 53, 2015, Pages 199-207, ISSN 1877-0509, http://dx.doi.org/10.1016/j.procs. 2015.07.295
Andranik Tumasjan, et al. 2010. Predicting Elections with Twitter: What 140 Characters Reveal about Political Sentiment. In Proceedings of the Fourth International AAAI Conference on Weblogs and Social Media. (Washington, DC, USA, May 23-26, 2010).

Chunyang Wang, Hailin Qu, Maxwell K. Hsu, Toward an integrated model of tourist expectation formation and gender difference, Tourism Management, Volume 54, 2016, Pages 58-71, ISSN 0261-5177, http://dx.doi.org/ 10.1016/j.tourman.2015.10.009

Yang Liu, Xiangji Huang, Aijun An, and Xiaohui Yu. 2007. performance using blogs. In Proceedings of the 30th annual international ACM SIGIR conference on Research and development in information retrieval (SIGIR '07). ACM, New York, NY, USA, 607-614. DOI= https://doi.org/10.1145/1277741.1277845

Carlos Raymundo, Cesar Mérida, Richer Rios and Alfred Kobayashi, 2017, Modelo arquitectónico de información para una plataforma de Big Data para el Sector Turístico. In Proceedings of the Décima Sexta Conferencia Iberoamericana en Sistemas, Cibernética e Informática (CISCI 2017), Orlando, Florida, EE.UU.

Anon. 2017. Big Data and Business Analytics Revenues Forecast to Reach $\$ 150.8$ Billion This Year, Led by Banking and Manufacturing Investments, According to IDC. (March 2017). Retrieved June 10, 2017 from http://www.idc.com/getdoc.jsp?containerId=prUS4237 1417. ARSA: a sentiment-aware model for predicting sales 